\title{
Direct Observation of the Behavior of a Single Gold Grain Boundary by In Situ TEM
}

\author{
Gilberto Casillas, Arturo Ponce, J. Jesús Velázquez-Salazar, Miguel Jose-Yacaman \\ Department of Physics and Astronomy, University of Texas at San Antonio, \\ One UTSA Circle, San Antonio, Texas 78249, USA.
}

Direct studies of nanostructures in situ of properties of individual nanostructures are not so common in the literature; nonetheless, they are increasing in number due to the development of new in situ holder for electron microscopes where it is possible to probe individual nanostructures (e. g. nanoparticles, nanowires, etc.) while observing the dynamics of the physical or chemical processes in real time. In-situ TEM studies have enabled the ability to observe dynamical process during mechanical deformation in polycrystalline thin films [1], single metal-metal contacts of $\mathrm{Ag}$ and $\mathrm{Au}$ by a cold-welding process assisted by an applied bias. Moreover, a cold-welding technique without an applied bias was also reported by $\mathrm{Lu}$ et al. [2] where they were able to weld ultrathin $\mathrm{Au}$ nanowires due to a similar crystallographic orientation between nanowires. A more detailed study of $\mathrm{Au}-\mathrm{Au}$ contacts was done by Merkle et al. [3] where they observed a liquid-like behavior between the gold contacts, resulting in necking after pulling or shearing of the contacts.

Here we report in situ HRTEM studies of a single grain boundary (GB) formed between gold nanoparticles formed by a cold-welding process. Briefly, colloidal gold nanoparticles were placed on the tip of a $0.2 \mathrm{~mm}$ gold wire, which then was mounted to an AFM-TEM holder from Nanofactory, Inc. The in situ experiments were carried out in a JEOL 2010F equipped with an ultra high resolution pole piece $(0.19 \mathrm{~nm}$ point resolution). Once in the microscope, the Si tip is used to "pick up" a nanoparticle and then bring another nanoparticle into contact with the first one.

Figure 1 shows a TEM sequence of the formation, neck growth and migration of the GB by mechanical manipulation of the bottom nanoparticle. By measuring the neck growth over time it was possible to estimate the surface diffusion coefficient between the nanoparticles. Moreover, the GB was able to rotate clockwise and counterclockwise several times in a liquid-like manner. The controlled movement of the nanoparticle revealed that free surfaces allow the free rotation of the GB despite crystallographic orientation.

Figure 2 shows a sequence of the same particles as in Figure 1 where GB sliding and GB migration is observed. In Figure $2 \mathrm{~b}$ the bottom left particle is moved to the upper left position until GB sliding is observed, leaving two new free surfaces; which after moving back the nanoparticle will be covered by the GB again. Moreover, we can observe shrinkage of the bottom nanoparticle from Figure $2 \mathrm{a}$ to $2 \mathrm{f}$, which is due to the migration of the GB.

Rotations of 90 of a GB were observed, as well as creation of new grains (which disappeared rather quickly due to the small size) due to rotations unfavorable to the crystal planes, as well as plastic deformation by slip of closed packed planes. Finally, this methodology can be used to study any kind of nanoparticles, such as semiconductors, metals, oxides, ceramics, or a combination of them, allowing a detailed study of the properties (e.g. sintering, surface diffusion coefficient) at the nanoscale of any system [4]. 


\section{References}

[1] F. Mompiou, M. Legros and D. Caillard, J. Mater. Sci. 46 (2011), p. 4308.

[2] Y. Lu, et al., Nat. Nano. 5 (2010), p. 218.

[3] A. P. Merkle, L. D. Marks, Wear 265 (2008), p. 1864.

[4] The research was supported by the NSF-PREM Grant \# DMR 0934218 and the Grant \# DMR1103730. The authors would also like to thank the Welch Foundation grant award \# AX-1615 and the NIH - RCMI: RCMI grant 5G12RR013646-12.
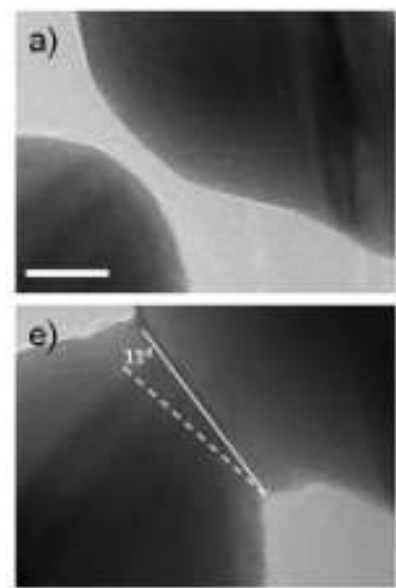
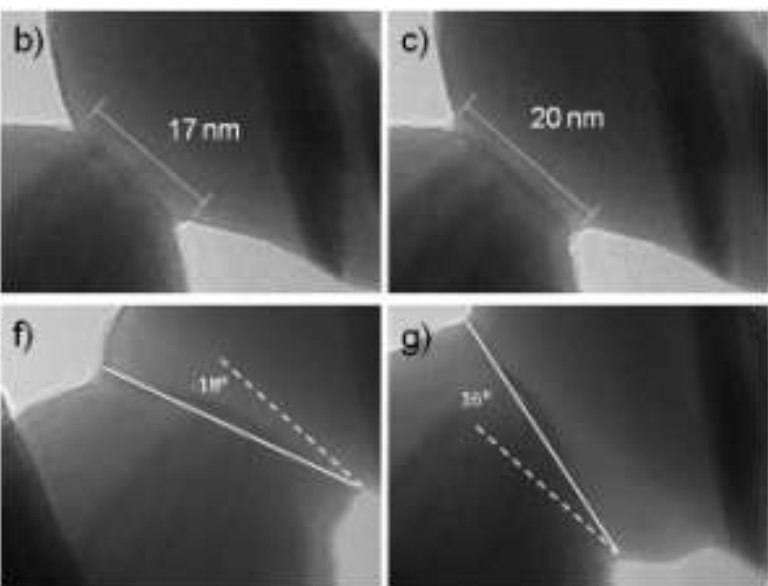

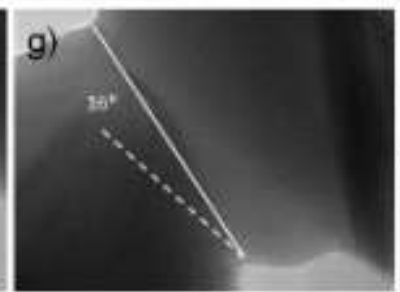

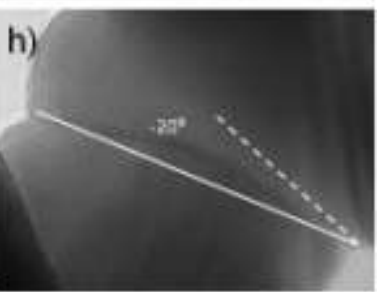

Figure 1. (a) Particles before contact at $0 \mathrm{~s}$. (b) Neck region at $17 \mathrm{~nm}$ in size at $1.33 \mathrm{~s}$. (c) Neck region $20 \mathrm{~nm}$ in size at 2.733. (d) Neck region $24 \mathrm{~nm}$ in size at $12.466 \mathrm{~s}$. Dashed line in (d) is used as a reference. The GB is rotated $11^{\circ}(\mathrm{e})(16.333 \mathrm{~s}),-18^{\circ}(\mathrm{f})\left(19.733 \mathrm{~s}, 16^{\circ}(\mathrm{g})(27.4 \mathrm{~s})\right.$ and $-20^{\circ}(\mathrm{h})$ $(36.73 \mathrm{~s})$ back and forth without breaking. Scale bar is $10 \mathrm{~nm}$.
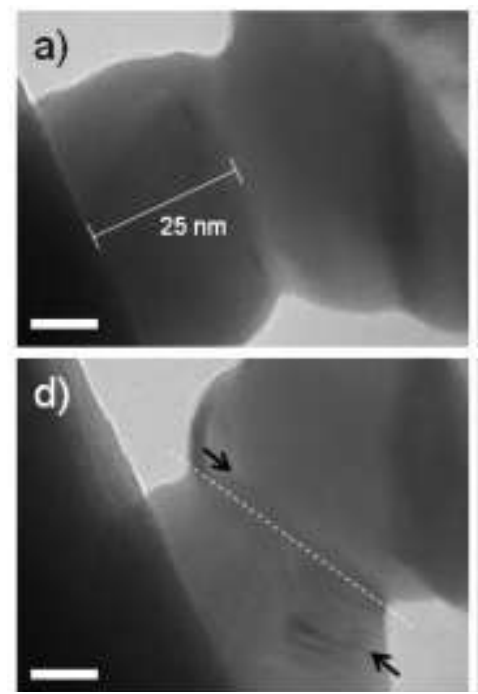
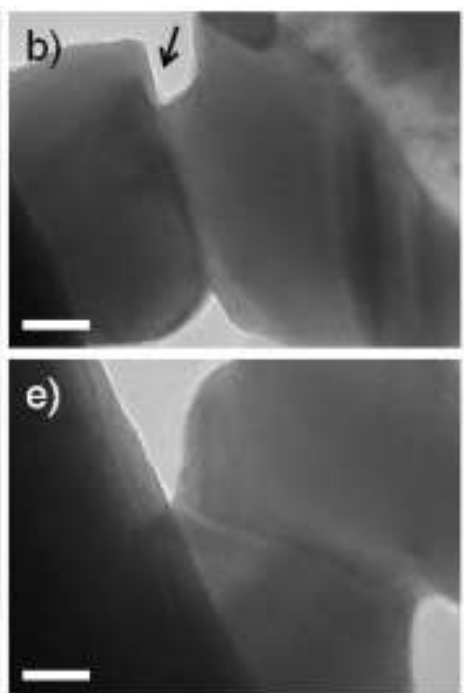
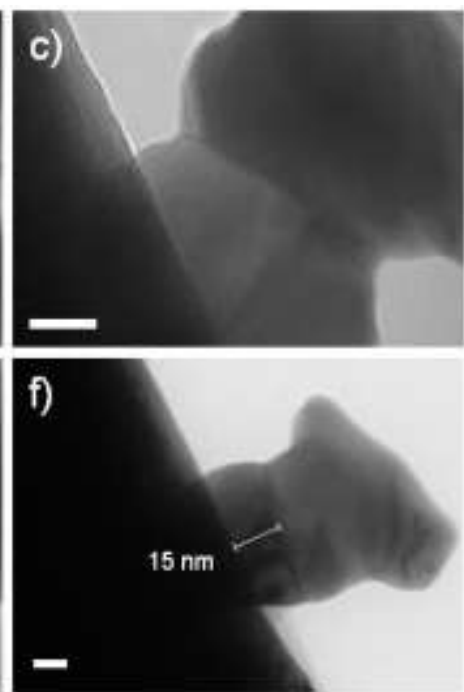

Figure 2. (a) GB at $69.266 \mathrm{~s}$. (b) The GB slid leaving a surface of the bottom -left particle (marked by an arrow) (70.866 s). (c) The GB is moved back covering the free surface left open in (b) and rotates counterclockwise $(76.666 \mathrm{~s})$. (d) The bottom particle is moved towards the beam direction and contrast fringes can be observed as the GB moves(marked by arrows) $(80.8 \mathrm{~s})$. (e) The GB is moved back to an almost on-edge view (84.466 s). (f) The nanoparticles detached from the Si tip. (120 s). Scale bars are $10 \mathrm{~nm}$. 